\title{
LAS PARADOJAS DE LA AUTORIDAD
}

\section{Paul Ricoeur ${ }^{(1)}$}

La autoridad, es preciso reconocerlo, tiene mala prensa en nuestros días. Se ha vuelto casi imposible una defensa pura y simple de la autoridad. ¿Qué nos queda, entonces?

Lo que propongo es el redescubrimiento de las cuestiones ineluctables que tiende a disimular una concepción no dialéctica de la autoridad, en la medida en que se ofrece como una respuesta que suprime la cuestión. Para reconquistar la dimensión problemática perdida, propongo la siguiente paradoja inicial:

Por un lado, quien autoriza viene de más lejos que quien es autorizado. Pero, por otro lado, la autorización no procede sin una relación más disimulada de reciprocidad. Esta primer paradoja de la reciprocidad en la disimetría abre el campo a una secuencia de variaciones que exploraremos. Todas ellas revierten sobre ese punto opaco: algo más elevado autoriza a la autoridad, pero ese algo que autoriza no vale si no es reconocido; existe una relación de retorno que hace de la autorización, a la vez, lo fundado y lo fundante.

Antes de desplegar esta paradoja quisiera decir algunas palabras sobre dos tradiciones filosóficas importantes, que domi-

\footnotetext{
(1) Traducción del francés de Aníbal Fornari.

Este artículo. originalmente leído en el Coloque "Morale" el 15 de enero de 1994 como homenaje a H-G. Gadamer en sus 90 años fue, entre otros más. solicitado a P.Ricoeur y preferencialmente concedido para Tópicos, que agradece profundamente al autor.
} 
naron la filosofía moral y que parecerían ahorrarse la noción de autoridad y de sus enigmas, en la medida en que tanto la una cuanto la otra ubican la fuente de la autoridad lo más cerca posible del agente de la acción, por ende lo más cerca de nosotros mismos. Pienso ante todo en Aristóteles cuya ética está enteramente enrraizada en el deseo razonable, en la aspiración deliberada a una vida plena, a una vida gozosa. La cuestión está en saber si esa aspiración es capaz de asegurar la plena autarquía del sabio. Pienso luego en Kant cuya idea-clave en filosofía moral es la autonomía, es decir la tesis de la identidad entre sí-mismo y la norma. Todo lo que no es autonomía, por tanto toda dependencia de un otro, debe ser dejada a un lado como heteronomía. La autoridad aparece así marginada del lado de la heteronomía. Pregunta: ¿Acaso nada habría que decir a favor de la autoridad sin agraviar a la autonomía?.

\section{$-\mathbf{I}-$}

Para responder a la cuestión planteada por cada una de estas grandes filosofías morales considerarétres temas que me parecen, en su conjunto, constituir el corazón opaco de la idea de autoridad. Yendo de lo más simple a lo más difícil, hablaré ante todo del sentido de la exterioridad ligado a la idea de autoridad. Mientras en Aristóteles el deseo aparece como constitutivo de nuestra interioridad de vida y de acción, y que en Kant la razón práctica aparece como interior a sí misma, la primera manifestación de la exterioridad está en la irrupción del otro en el campo moral. Según Levinas, del otro nos viene la prescripción de lo que él denomina el llamado a la responsabilidad. De tal modo, mantener su promesa no es simplemente ponerse de acuerdo consigo mismo, por temor a renegarse, sino también responder a la espera del otro: alguien cuenta conmi- 
go, cuenta con que mantendré mi promesa y me ayuda obligándome. La primer idea que retendremos es, entonces, la de una exterioridad solidaria con la idea de alteridad.

La segunda idea a considerar es la de superioridad. Con ella reencontramos nuestra paradoja inicial, captando la dimensión vertical propia de la relación mandar-obedecer. Tocamos ahí una experiencia de la conciencia moral que encuentra su expresión en figuras de la autoridad. La autoridad se figura. Toma figura en los maestros griegos de sabiduría, en los maestros judíos de justicia; generalizando, puede decirse que, probablemente, no habría vida moral sin la ejemplaridad de los grandes testigos de la vida moral. Insisto sobre la palabra ejemplaridad, que incluye un elemento de larga duración y, en consecuencia, no se deja confundir con lo inmutable, como es aún el caso de la encíclica pontifical Veritatis Splendor, donde se pasa demasiado rápidamente de la ejemplaridad de Cristo a la inmutabilidad de la regla moral. La ejemplaridad, me parece, comporta una dimensión histórica en la medida en que incluye la capacidad de afrontar la historia, de engendrarla y, por eso, de escapar a la alternativa entre lo inmutable y lo cambiante, alternativa en la que la discusión moral se empantana a menudo, como si no hubiese sino dos soluciones: o reglas eternas, inmutables, o una historia inconstante y cambiante de la conciencia moral. Inversamente a esta alternativa, la ejemplaridad comporta un elemento temporal de perennidad, que no significa ausencia de historia sino, si puede decirse, resistencia en la historia. Este trazo notable es esencial a este segundo componente de la idea de autoridad, es decir, al elemento de superioridad.

La tercer idea constitutiva, luego de exterioridad y de superioridad, es la de anterioridad. Aquí es donde nuestra perplejidad es mayor. En efecto, nunca podemos estar seguros sobre el momento de nacimiento de una norma. Podemos rebelarnos contra 
las reglas, podemos también cambiarlas -lo que es esencial al carácter histórico como contrapartida de lo que se acaba de decir sobre la perennidad-, pero ante todo hay herencia, ante todo hay deuda. Tocamos aquí una extraña paradoja que nos lleva al corazón de la dificultad: siempre hay una ley anterior a la ley. Antes de la leyes existe la Ley, esto es, todo el conjunto del campo simbólico constitutivo de nuestra humanidad y que, a tal título, convierte a la ley en una instancia siempre anterior a sí misma.

Tal paradoja de la vida moral está en paralelo con otros campos adyacentes al de la moralidad. Pienso primero en el orden político. En realidad la idea de autoridad tiene su lugar de nacimiento en el campo político. Aquí es donde se da claramente el fenómeno de la jerarquía: arriba los gobernantes, abajo los gobernados. Todo el análisis de lo político hecho por Max Weber comienza con el surgimiento de esta relación jerárquica dentro del campo social. Por eso la idea de autoridad se confirma como inexpugnable. Puede evocarse al respecto la formula latina que Hannah Arendt se complace en repetir: potestas in populo, auctoritas in senatu: el poder está en el pueblo, la autoridad en el senado. Lo que significa que el poder político se sitúa en el cruce de dos ejes, uno vertical y el otro horizontal. Por un lado, el poder es lo que simplemente emerge del querer vivir juntos; existe una comunidad histórica tanto tiempo cuanto la gente quiera vivir bien junta. Cuando ya no lo quiere, el vínculo cívico queda vencido(2), en el sentido fuerte de la palabra vencido: se puede hablar de quedar vencido históricamente como se habla de una derrota militar. Ese espec-

\footnotetext{
(2) El Autor hace oscilar semánticamente el verbo défaire= des-hacer. destruir: "le lien civique se défait $=$ se deshace". y la substantivación "au sens fort du mot dé-faite" =derrota. destrucción. Traducimos vencer, pues mantiene la oscilación entre el haber terminado su vida útil un producto. una relación contractual o un tejido vinculante. $y$ el ser militarmente vencido. (Nota del Traductor).
} 
táculo trágico de vencimiento lo dan hoy pueblos enteros en la exYugoslavia o en la ex-Unión Soviética. Pero el poder no adquiere duración si el eje horizontal no cruza el eje vertical de la autoridad. ¿En qué consiste, entonces, esa auctoritas que los latinos ubican en el Senado? Es claro que el pueblo existe, ahora, en la medida en que puede reunirse en el foro, en el ágora. ¿Pero el Senado? ¿Es, acaso, la Asamblea de los Ancianos? En un sentido, sí. Pero esta localización es, en otro sentido, engañosa. En efecto, si se busca el origen histórico de lo que Max Weber llama dominación (Herrschaft), uno se encuentra tomado por una especie de huida hacia atrás: jamás se encuentra un primero. Previamente a un imperium hay otro imperium. Como si un Cesar naciera siempre de otro Cesar, así fuera democrático. La cadena de autoridad se pierde en la noche de los tiempos. Y esto es tan cierto que todo nuevo poder trata de legitimarse como renacimiento, recurriendo a un poder más antiguo que él. Michelet pinta a la Revolución Francesa como tiempo cero. Pero tal tiempo no se pensó a sí mismo como tiempo cero, sino que se pensó como recuperación de la República romana. Sólo hay renacimiento donde hay repetición de una autoridad anterior. ¿Quién es, entonces, el primer Cesar? Los Cesar, decimos, nacen de los Cesar, pero tras los Cesar hay un Alejandro, y tras Alejandro están los potentados orientales ... Nunca uno se topá con un primer poder.

\section{-II-}

Se puede ahora intentar transportar este análisis desde el campo lateral de lo político hacia el campo moral mismo. ¿Qué es lo que significa, entonces, esta relación de precedencia en el campo moral? Significa esto: las grandes instancias morales y las grandes 
costumbres morales se agregan siempre a una fundación que equivaldría en el orden moral a lo que es la auctoritas en el orden político. Este problema fue reencontrado por numerosos filósofos bajo la cuestión del legislador. Así es como Rousseau, partiendo de la tesis de que la voluntad general no se reduce a la voluntad mayoritaria, a la suma de voluntades individuales, es compelido a interrogarse de qué modo esta voluntad general puede vincularse con nuestras voluntades individuales. Lo que él propone es que tal vinculación no es posible sino a través de la ayuda de un mediador, a saber, el legislador. Con la cuestión del legislador nos topamos con una figura mayor de la autoridad: la de la autoridad autorizada. Se trata de una paradoja puesto que es el legislador mismo quien recibe la ley. En nuestra tradición occidental judeocristiana Moisés es la figura emblemática de tal paradoja. Contamos aquí con la figura misma del legislador, quien no es legislador de su pueblo sino en cuanto recibe la ley. Todos los relatos tradicionales referidos al Sinaí merodean en torno a este nudo: Moisés recibe las Tablas que son primero quebradas, luego reescritas y, finalmente, perdidas. Pero el origen permanece siempre hacia atrás. Se reencuentra así, en el plano moral, un enigma completamente paralelo al evocado hace instantes en el orden político, donde el legislador está siempre precedido por otro legislador.

Podría hacerse otra comparación, esta vez con el orden cósmico. Siempre hubo vinculación entre la idea de creación y la idea de legislación, ya sea en el ámbito bíblico o en el pensamiento griego, como puede observarse en los Estoicos, quienes magnifican el parentesco entre el orden cósmico y el orden moral. Nuestra tradición judeocristiana presenta un enigma del todo semejante a los relatos de creación que nunca nos tornan contemporáneos de un inicio absoluto. Al respecto, la estructura de estos relatos de creación es sumamente interesante si se la compara con los relatos 
de legislación, como la legislación de Moisés en el Decálogo. Tampoco ahí se puede hacer coincidir el origen con un comienzo. Se intenta fechar y cercar el comienzo, pero en una regresión sin fin. El origen está ya siempre ahí, huyendo hacia atrás. De tal modo, los relatos de creación intentan en vano hacer coincidir el origen con el comienzo. Hay, entonces, una suerte de dialéctica entre el origen y el comienzo: al comienzo se lo puede tratar de fechar, pero el origen es siempre anterior. Así es cómo ya no estamos más en posesión de un comienzo de la vida moral, como no lo estamos de un comienzo del orden político ni del orden cósmico.

Ahora podemos volver a nuestras dos grandes tradiciones de filosofía moral, antes evocadas, la de Aristóteles y la de Kant. Puede verse allí el intento de eludir el problema de lo que podría denominarse la fundación fugaz ${ }^{(3)}$, esa fundación previa a la fundación.

En Aristóteles, donde el principio ético parece residir en la intimidad del sujeto voluntario, la idea de exterioridad retorna en los grandes capítulos sobre la amistad y la justicia. El sujeto no se funda sobre sí mismo sino en la medida en que recibe del otro la autorización de existir, en una relación de amistad y de justicia. En esta relación de reconocimiento por el otro es donde se construye la identidad personal. La alteridad se insinúa así en el corazón de la intimidad. La intimidad y la exterioridad van a la par en relación a la amistad y la justicia. Pero reencontramos también el tema de la superioridad ligada al deseo de la vida buena; pues nadie engendra, en su propio deseo, la estructura misma de las virtudes que mediatizan ese deseo. En toda la tradición antigua esos modelos de

\footnotetext{
(3) Cursivas nuestras. por la importancia que la categoria adquiere en este texto y en el conjunto del pensamiento de Ricoeur, expresando la apertura y la discreción de la fundamentación, en tanto es el intento de articular, dentro del discurso, al punto de fuga' real. (Nota del T.).
} 
vida plena tienen nombre. Está la figura del coraje, la figura de la amistad, de la generosidad, etc. Todo el orden ético es así atraído hacia lo alto por una exterioridad-superioridad que arranca al deseo y lo eleva a una altitud superior a sí mismo. De este modo, es muy difícil disociar, en Aristóteles, el análisis de la vida moral que reposa sobre el deseo de la plenitud, de las configuraciones de la perfección, de las virtudes, en lenguaje aristotélico, las que están atravesadas por un movimiento ascendente que subordina los valores morales a los valores políticos, y éstos a los valores de la contemplación. Esta atracción desde lo alto es constitutiva de la vida ética, cuya organización propia no existe sin la afinidad con un orden cósmico que gravita en torno a un Dios que atrae hacia sí sin mandar.

Pero Kant es quien queda como el caso más difícil. ¿Pudo, acaso, extirpar de la idea de autonomía todo componente de exterioridad, de superioridad, de anterioridad, sin que ellos caigan bajo la idea de heteronomía? Veamos. Consideremos la segunda formulación del imperativo categórico, que prescribe tratar a la humanidad en nuestra persona y en la del otro como un fin en sí y no sólo como un medio. Una especie de exterioridad se perfila aquí, la de la humanidad figurada por la otra persona. Así es como una suerte de exterioridad, la de la relación interpersonal, prevalece sobre el más íntimo imperativo. La idea de heteronomía se presenta aquí demasiado corta, como recién lo fue con la amistad o la justicia según Aristóteles. Éstas nos colocan bajo la ley del otro, según la ley de la espera del otro respecto a nosotros. Por lo demás, en el mismo Kant la definición de autonomía comporta una zona de sombra, en la medida en que la autonomía es, radicalmente, la ley que uno de da a sí mismo. Ahora bien, esta síntesis de la libertad y de la ley es presentada por Kant como un "hecho de la razón", extraño a toda deducción desde un principio previo como también a toda verifica- 
ción por la historia. La estructura del imperativo es, de algún modo, "dada" y, por eso, siempre es ya ahí. Al respecto puede pensarse que el criterio de universalidad de las máximas de la voluntad constituye sólo una regla de control y en modo alguno es un principio de fundación última. Finalmente, cuando Kant se halla frente a la cuestión del mal, es conducido a meditar sobre las condiciones de una regeneración de la voluntad en su libre arbitrio. Aquí se coloca una cuestión totalmente diferente a la de la autonomía, a saber, cómo una voluntad puede ser convertida en apta para llegar a ser la voluntad de un sujeto moral. Pues bien, ¿cómo llegaría a serlo si no fuese devuelta a su autonomía mediante la gracia de una ayuda exterior, que carece de sentido si ella no se inscribe en un proceso de regeneración de un querer capaz de autonomía?.

\section{-III-}

Quisiera proponer, en esta última parte de mi contribución, una solución dialéctica de la autoridad que ayude a pensar lo exterior, lo superior, lo anterior, es decir, los tres predicados que hemos encontrado adheridos a la idea de autoridad. La cuestión reside en saber cómo puede hacerse de la autoridad, no ya lo contrario de la autonomía, sino una íntima incitación a la autonomía, un llamado a la autonomía, un componente, en fin, que no sólo fuese compatible con la autonomía, sino que simultáneamente la exigiera, la acompañara, más aún, la completara.

Retomemos uno tras otro a los tres predicados, cual trazos característicos de la idea misma de autoridad.

¿Qué sucede con la exterioridad en una perspectiva más dialéctica que la de la dicotomía entre autonomía y heteronomía? 
El punto decisivo está en que la exterioridad de la incitación a la autonomía no funciona sin reconocimiento. Ahora bien, no hay reconocimiento sin mutualidad. En este sentido, el concepto más importante a introducir aquí es el de reconocimiento mutuo. A su vez, la superioridad de la ley carece de sentido si también ella no es reconocida, tal como es manifiesto en la experiencia de la "voz" de la conciencia moral. Hay ahí un vínculo muy sutil: a la superioridad misma responde el reconocimiento de superioridad. Este es el segundo empleo de la noción de reconocimiento. Se halla esta paradoja encarnada en la relación de instrucción constitutiva de la relación entre maestro y discípulo. Esta relación ciertamente comporta un aspecto jerárquico, pero, simultáneamente, el superior no es tal sino en tanto es reconocido.

¿Qué sucede, finalmente, con la anterioridad? Podría repetirse por tercera vez lo que acaba de decirse sobre el rol del reconocimiento: si cabe decir que hay anterioridad del llamado del otro a la responsabilidad, es la respuesta-responsabilidad-a tal llamado lo que restituye la mutualidad y una cierta igualdad entre el apelante y el apelado. Pero es más instructivo introducir una nueva paradoja, a saber, que la anterioridad misma de la ley es inseparable del trabajo de interpretación que la actualiza. Todo el orden simbólico no se torna productivo sino mediante reescrituras incesantes de la ley, las que llegan a justificar a posteriori el a priori de la anterioridad. De este modo puede afirmarse que hay exigencia de autonomía hasta en la anterioridad de la ley.

Para darle cuerpo a esta paradoja, permítaseme buscar un primer apoyo en un filósofo judío, Franz Rosenzweig, autor de un libro extraordinario, La Estrella de la Redención. Hablando de la ley este autor explica que ella implica en su sentido mismo algo que le es anterior y que él denomina el mandamiento. Este mandato es único: "Tú amarás a tu prójimo como a ti mismo". Dicho de otro 
modo: previamente a las leyes existe el mandamiento de amar. Ahora bien, aquí está el más extraño de los mandamientos, pues ¿cómo uno puede mandar a un sentimiento? Para responder a esta esperada objeción es preciso decir que este mandato del amor es algo bien distinto de una emoción; es una disposición de la voluntad y del ser entero; además -y esto es lo más importante- el mandamiento mismo es el estatuto de una obligación constituyente. Tal estatuto puede ser resumido en una fórmula: fundamentalmente, el amor es fuente de obligación, el amor obliga. Para comentar el imperativo de amar apelaré a otro pensador judío, Hans Jonas, según el cual aquello a lo que estamos fundamentalmente obligados es a socorrer al frágil. De quien es frágil somos esencialmente responsables. Tenemos una experiencia familiar simple de este vínculo: desde que un niño nace nos obliga a hacerlo crecer, a ayudarlo a volverse grande. Estamos aquí sobre un terreno que puede ser compartido por creyentes y no-creyentes. El creyente dirá junto al apóstol Juan: "Dios es amor". Pero podemos también nombrar a Eros al modo de Platón, aún a la manera del Freud de los últimos escritos. El amor es la capacidad de recorrer en ambos sentidos, ascendente y descendente, la escala de lo erótico, de la amistad y de la caridad. En esta relación entre tres figuras del amor es donde nace la obligación. Tal es el modo indirecto por el que podemos aproximarnos al enigma de la anterioridad. Ese siempre-ya-ahí de lo que nos ha obligado es lo que constituye el corazón del enigma y la opacidad de la idea de autoridad. Y lo que ya desde siempre nos ha obligado es el amor, sea que lo personifiquemos en un ser, sea que hagamos de él una fuerza psíquica o una energía cósmica. La fórmula: "el amor obliga", me parece ser la más apta para designar el punto de sutura entre el orden fundador y el orden fundado. Podría irse hasta esta fórmula: a lo que el amor obliga es a lo que me gustaría denominar obediencia amante. 
A este precio puede decirse que la autoridad exige la autonomía. Insisto sobre el verbo exigir: el amor no es al respecto un substituto de la justicia, sino una demanda de más justicia, una exigencia de lo que es más en la justicia. Puesto que el amor es, ante todo, exceso, puede por eso mismo pedirle a la justicia ser más plenamente justicia, serla hasta el fondo, a pesar de lo que cueste. Tras este rodeo volvemos a Kant, en tanto el amor pide, ante todo, que la justicia sea verdaderamente universal y no reduzca su radio de acción a los límites del clan, del grupo étnico, etc. Pasando por el exceso de lo que es casi imposible, lo universal es reclamado a ser él mismo. Pero al mismo tiempo, la justicia es reclamada a ser más singularizante, es decir, atenta al carácter insubstituible de las personas. Esta otra cara de la exigencia de un más en justicia es resultante de la dialéctica entre la norma y la persona: la solicitud va a las personas a través de la norma. Ahí es donde la justicia debe hacerse equidad, esto es, atenta a la singularidad del frente a frente en la relación de justicia.

Pero puede también decirse que la autoridad del amor no sólo al comienzo exige la justicia, sino que también la acompaña, invitando a la autonomía a inscribirse en una relación verdaderamente mutua. El camino que usaré para expresar esto es el de Habermas, en su voluntad de no dejar que la ética de la acción comunicativa se deje subyugar por una argumentación estratégica o utilitaria. Es preciso, entonces, que la razón comunicativa sea realmente más fuerte que la razón instrumental para que en nuestra idea de justicia prevalezca el sentimiento de un mutuo endeudamiento, por sobre el de un interés recíproco de preservarnos los unos de los otros, tal como parece ser todavía el caso de una concepción demasiado limitada de la justicia que consistiría en decir: aquí termina tu imperio, ahí comienza el mío y, entre ambos, coloquemos el límite de cada uno cabe sí mismo. Frente a la amenaza 
resultante de una total fragmentación del vínculo social, la idea de justicia no puede ya ser mantenida en su más alto nivel sino elevándose al sentimiento de un endeudamiento mutuo.

Para concluir diré que en este llamamiento a un mutuo endeudamiento es donde la precedencia de la autoridad llega a expresarse en el corazón de la inmanencia del querer consigo mismo y en lo cotidiano de las jornadas. 